\title{
Photonic spectrum of bichromatic optical lattices
}

\author{
Stefan Rist ${ }^{1}$ Patrizia Vignolo ${ }^{2}$, and Giovanna Morigi ${ }^{1}$ \\ ${ }^{1}$ Grup d'Òptica, Departament de Física, Universitat Autònoma de Barcelona, 08193 Bellaterra, Spain \\ 2 Institut Non Linéaire de Nice, Université de Nice-Sophia Antipolis, \\ CNRS, 1361 route des Lucioles, 06560 Valbonne, France
}

\begin{abstract}
We study the photonic spectrum of a one-dimensional optical lattice possessing a double primitive cell, when the atoms are well localized at the lattice minima. While a one-dimensional lattice with a simple Wigner-Seitz cell always possesses a photonic bandgap at the atomic resonance, in this configuration the photonic transmission spectrum may exhibit none, double or multiple photonic bandgaps depending on the ratio between the interparticle distance $\varrho$ inside the cell and the cell size $a$. The transmission spectra of a weak incident probe are evaluated when the atoms are trapped in free space and inside an optical resonator for realistic experimental parameters.
\end{abstract}

\section{INTRODUCTION}

Ultracold atoms in optical lattices constitute a paradigmatic system, which allows one control over several parameters, thereby mimicking dynamics typical of condensed matter systems [1, 2]. A remarkable feature of optical lattices is that the bulk periodicity is here controlled by engineering the geometry of the propagating beams, which determine the light potentials. Differing from ordinary crystals in condensed matter, the size of the Wigner-Seitz cell is of the order of the light wavelength. One consequence is that the light, coupling with the atomic transitions, is also diffracted by the crystallyne structure which the atoms form [3].

It has been observed that the modulation of the atomic density in these systems, and hence of the refractive index, makes optical lattices a photonic bandgap material [4]. Theoretical works studied the photonic bandgap for one-dimensional and three-dimensional atomic structures [4, 5, 6, 67, 8, 9]. Besides potential applications for nonlinear optics, the full understanding of these properties is important in order to measure the quantum state of cold atoms using light [10], thereby opening interesting avenues in quantum information processing for implementing photonic interfaces using ultracold atoms [11].

In this work we study theoretically the photonic properties of biperiodic optical lattices, in a setup similar to the ones realized experimentally in [12, 13, 14]. We focus on a one-dimensional configuration, and develop a full quantum model for the light-matter interactions, assuming that the atoms are well localized at the lattice minima. The photonic spectra and the probe transmission are evaluated when the optical lattice is in free space and inside a standing wave optical resonator, as a function of the interparticle distance $\varrho$ inside the primitve cell.

This article is organized as follows. In Sec. II the theoretical model is described and the basic approximations are introduced. In Sec. III the photonic spectra are reported and discussed, and the transmission spectra for a weak probe are evaluated in Sec. IV. In Sec. V the photonic properties of bichromatic optical lattices inside a resonator are analyzed. The conclusions are presented in Sec. VI

\section{THEORETICAL MODEL}

The physical system we consider is a one-dimensional periodic distribution of atoms in a light potential with a double primitive cell. We assume a sequence of $N$ atoms of mass $m$ in a standing wave created with lasers along the $x$ direction. We denote the atomic positions along $x$ by $x_{j}$, with $j=1, \ldots, N$. Denoting by $a$ the size of the Wigner-Seitz cell, the positions are given by

$$
\begin{aligned}
x_{j} & =\ell a \text { for } j=2 \ell, \\
& =\ell a+\varrho \text { for } j=2 \ell+1,
\end{aligned}
$$

where $\ell=0,1, \ldots, M-1$, and $M=N / 2$ is the number of cells (assuming $N$ even for convenience). In the case here discussed, we set the size $a=\lambda$, where $\lambda$ is the wavelength of the light which interacts with the dipolar transitions of the atoms.

Such configuration can be experimentally realized by using a monochromatic standing wave with wavelength $\lambda$, to which two laser beams are superposed, such that they are rotated by angles of $60^{\circ}$ and $120^{\circ}$ with respect to the axis of the lattice, as shown in Fig. 1. Upon setting the relative phases, the resulting potential for the atoms has the form

$$
U(x) \propto \beta^{2} \cos ^{2}(k x / 2)+\cos ^{2}(k x)
$$

and the distances between adjacent wells are $d_{1}=\lambda(1-$ $\frac{1}{\pi} \operatorname{acos} \frac{-\beta^{2}}{4}$ ) and $d_{2}=\frac{\lambda}{\pi} \operatorname{acos} \frac{-\beta^{2}}{4}$, with $d_{1}+d_{2}=\lambda$. Another possible realization is found by superposing two laser beams along the $x$-axis, with a half frequency [15] or with a three fourth frequency respect to the frequency of the main lattice [16]. Upon setting the relative phases, the four-atomic elementary cell has the structure $d_{1}-d_{1}-d_{2}-d_{2}$ (with $d_{1}+d_{2}=\lambda$ ) and the crystal has essentially the same spectral properties than the biatomic one considered in this paper. In this work we will also consider biperiodic lattices, where the atoms composing the Wigner-Seitz cell may have different scattering properties, for instance, they can belong to different species 


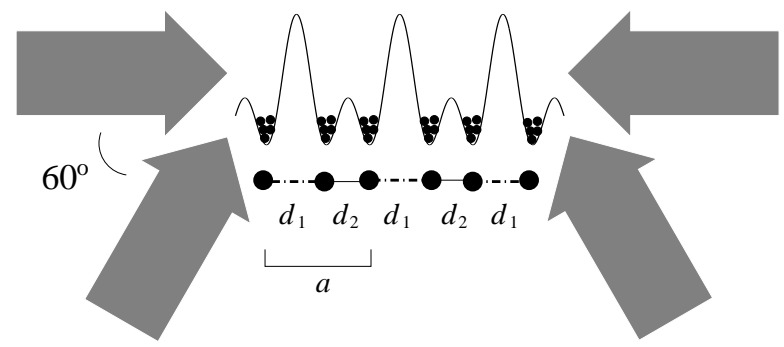

FIG. 1: A possible optical realization of the double period $1 \mathrm{D}$ lattice, considered in this work, can be obtained by using a monochromatic standing wave with wavelength $\lambda$, to which two laser beams are superposed, such that they are rotated by angles of $60^{\circ}$ and $120^{\circ}$ with respect to the axis of the lattice. Here, $d_{1}=\varrho, d_{1}+d_{2}=\lambda$ and the size of the Wigner-Seitz cell is $a=\lambda$.

or belong to the same species but are prepared in different hyperfine states. Under the assumption that the frequencies of the two transitions are sufficiently close to allow significant coupling with the same probe, such lattices could be realized with linearly polarized counterpropagating beams, controlling the angle between the polarization [17].

In developing the theoretical model we will make the following assumptions: (i) The atoms are well localized at the lattice points, and the size of the atomic wave packet is very small with respect to the laser wavelength (LambDicke regime [18]). This situation can be realized when the atoms are deep in the Mott-insulator quantum state of the biperiodic potential [1]. We will treat the atoms as pointlike, hence considering the response of the medium at lowest order in the expansion of the size of the wave packet over the wavelength. (ii) We consider the coupling of the lattice only with modes of the electromagnetic field which propagate along the lattice. This approximation is valid when the atoms are placed, for instance, inside a bad cavity with sufficiently large cooperativity [19] or a hollow-core fiber [20, 21].

\section{A. Hamiltonian}

The Hamiltonian $H$ for the total system, composed by the atomic spins aligned along the $\hat{x}$ axis, and the modes of the electromagnetic field propagating along $\hat{x}$, reads

$$
H=H_{\mathrm{dip}}+H_{\mathrm{emf}}+H_{\mathrm{int}} .
$$

Here,

$$
H_{\mathrm{dip}}=\sum_{j} \hbar \omega_{j} \sigma_{j}^{\dagger} \sigma_{j}
$$

describes the array of dipoles, with $j=1, \ldots, N$ labeling the atoms, where $\sigma_{j}$ is the dipolar lowering operator and $\sigma_{j}^{\dagger}$ its adjoint, whereby the relevant transitions of the atoms at the even (odd) sites, $j=2 \ell(j=2 \ell+1)$, have dipole moments $\mathcal{D}_{1}\left(\mathcal{D}_{2}\right)$ and transition frequency $\omega_{1}\left(\omega_{2}\right)$. The Hamiltonian for the modes of the electromagnetic field takes the form

$$
H_{\mathrm{emf}}=\sum_{k} \sum_{n=1,2} \hbar \omega_{k} a_{k}^{(n) \dagger} a_{k}^{(n)}
$$

where the operators $a_{k}^{(n)}$ and $a_{k}^{(n) \dagger}$ annihilate and create a photon in the electromagnetic field mode at frequency $\omega_{k}$, wave-vector $k=\omega_{k} / c$, with $c$ light velocity, and polarization $\hat{\epsilon}_{n}=\hat{\epsilon}_{1}, \hat{\epsilon}_{2}$, with $\hat{\epsilon}_{1} \perp \hat{\epsilon}_{2} \perp \hat{x}$. Finally, the interaction between photons and atoms is described by the Hamiltonian term (in Coulomb gauge)

$$
H_{\mathrm{int}}=-e \sum_{j} \frac{p_{j}}{m_{j}} \cdot A_{\perp}\left(x_{j}\right),
$$

where $m_{j}$ is the mass of the atom $j, p_{j}$ is the momentum operator of the electron at atom $j$,

$$
p_{j}=\mathrm{i} \frac{m_{j}}{e} \omega_{j} D_{j}\left(\sigma_{j}^{\dagger}-\sigma_{j}\right),
$$

and $A_{\perp}(r)$ is the transverse vector potential

$$
A_{\perp}(r)=\sum_{k} \sum_{n=1,2} \sqrt{\frac{\hbar}{2 \varepsilon_{0} \omega_{k} V}}\left(a_{k}^{(n)} \hat{\epsilon}_{n} \mathrm{e}^{\mathrm{i} k \cdot r}+\text { H.c. }\right),
$$

with $\varepsilon_{0}$ the vacuum permittivity and $V$ the quantization volume. Note that we have used a plane waves decomposition, applying periodic boundary conditions at the lattice borders.

\section{B. Weak excitation regime}

In this work we consider that the atomic transitions are driven well below saturation, and correspondingly the mean number of photonic excitations inside the system is much smaller than the total number of spins $N$. In this regime we use the Holstein-Primakoff representation of spin operators [22], and expand all operators at the lowest orders in the powers of bosonic operators $b_{j}$,

$$
\begin{aligned}
\sigma_{j}^{\dagger} & =b_{j}^{\dagger}\left(1-b_{j}^{\dagger} b_{j}\right)^{1 / 2} \simeq b_{j}^{\dagger}\left(1-b_{j}^{\dagger} b_{j} / 2\right), \\
\sigma_{j}^{-} & =\left(1-b_{j}^{\dagger} b_{j}\right)^{1 / 2} b_{j} \simeq\left(1-b_{j}^{\dagger} b_{j} / 2\right) b_{j}, \\
\sigma_{j}^{z} & =-\frac{1}{2}+b_{j}^{\dagger} b_{j} .
\end{aligned}
$$

In this representation, the Hamiltonian for the dipoles becomes the sum of $N$ harmonic oscillators,

$$
H_{\text {dip }}=\sum_{j} \hbar \omega_{j} b_{j}^{\dagger} b_{j}
$$

where we discarded the constant term. The interaction term reads (in the Rotating Wave Approximation)

$$
H_{\mathrm{int}}=H^{(1)}+H^{(3)}
$$


with

$$
H^{(1)}=\sum_{j, k, n} \hbar \mathcal{G}_{j, k}^{(n)} b_{j}^{\dagger} a_{k}^{(n)} \mathrm{e}^{\mathrm{i} k x_{j}}+\text { H.c. },
$$

while $H^{(3)}$ describes the corrections beyond the linear response. Here, $\mathcal{G}_{j, k}^{(n)}$ is the coupling strength of the atom $j$ with the mode $(k, n)$ and is given by

$$
\mathcal{G}_{j, k}^{(n)}=-i \omega_{j} \mathcal{D}_{j} \cdot \hat{\epsilon}_{n} \sqrt{\frac{1}{2 V \varepsilon_{0} \hbar \omega_{k}}} .
$$

We will consider the limit in which we can truncate the expansion and approximate $H_{\text {int }} \approx H^{(1)}$, thereby restricting to the case in which the medium polarization is linear in the electric field amplitude.

\section{Spin waves}

Given the periodic structure, it is convenient to describe the dipolar excitations in momentum space. At this purpose, for a sufficiently large crystal we assume Born-von Karman periodic boundary conditions, and consider the spin-wave excitations

$$
\begin{aligned}
& b_{q}=\frac{1}{\sqrt{M}} \sum_{\ell=0}^{M-1} b_{2 \ell} \mathrm{e}^{-\mathrm{i} \ell q a} \\
& d_{q}=\frac{1}{\sqrt{M}} \mathrm{e}^{-\mathrm{i} q \varrho} \sum_{\ell=0}^{M-1} b_{2 \ell+1} \mathrm{e}^{-\mathrm{i} \ell q a}
\end{aligned}
$$

with $q$ the wave vector sweeping the first Brillouin zone (BZ). We denote by

$$
G_{0}=2 \pi / a
$$

the elementary vector of the reciprocal lattice, such that the interval of the first BZ is $\left[-G_{0} / 2, G_{0} / 2\right]$. Using the relation $\sum_{\ell=0}^{M-1} \exp \left(\mathrm{i}\left(q-q^{\prime}\right) \ell a\right)=M \delta_{q q^{\prime}}$ where the equality $q=q^{\prime}$ is defined modulus a vector $G$ of the reciprocal lattice, the Hamiltonian terms transform as

$$
\begin{aligned}
& H_{\mathrm{dip}}=\sum_{q \in B Z} \hbar\left(\omega_{1} b_{q}^{\dagger} b_{q}+\omega_{2} d_{q}^{\dagger} d_{q}\right) \\
& H^{(1)}=\sum_{G, n} \sum_{q \in B Z} \hbar \sqrt{M}\left(\mathcal{G}_{1, q+G}^{(n)} b_{q}^{\dagger}+\mathrm{e}^{\mathrm{i} G \varrho} \mathcal{G}_{2, q+G}^{(n)} d_{q}^{\dagger}\right) a_{q+G}^{(n)} \\
& \text { +H.c. }
\end{aligned}
$$

where the quasi-momentum verifies the relation $k=q+$ $G$. In this form, the Hamiltonian can be rewritten as the sum of $M$ Hamiltonian terms, $H=\sum_{q \in B Z} H_{q}$, where

$$
\begin{aligned}
H_{q} & =\hbar \omega_{1} b_{q}^{\dagger} b_{q}+\hbar \omega_{2} d_{q}^{\dagger} d_{q}+\hbar \sum_{G, n} \omega_{q+G} a_{q+G}^{\dagger(n)} a_{q+G}^{(n)} \\
& +\hbar \sum_{G, n}\left[\sqrt{M}\left(\mathcal{G}_{1, q+G}^{(n)} b_{q}^{\dagger}+\mathrm{e}^{\mathrm{i} G \varrho} \mathcal{G}_{2, q+G}^{(n)} d_{q}^{\dagger}\right) a_{q+G}^{(n)}+\text { H.c. }\right]
\end{aligned}
$$

This separation is only valid in the linear regime since saturation effects, described by $H^{(3)}$, mix the manifolds identified by the Hamiltonian terms $H_{q}$.

\section{THE PHOTONIC BAND STRUCTURE}

In this section we study the photonic spectrum of the biperiodic structure assuming that the polarization of the incident light, say $\epsilon_{1}$, is parallel to the dipole moments $\mathcal{D}_{1}$ and $\mathcal{D}_{2}$. Hence, we drop the polarization superscripts where they appear. The photonic band structure is found by solving the Heisenberg equations of motion for each Hamiltonian block $H_{q}$,

$$
\begin{aligned}
& \dot{a}_{q+G}=-\mathrm{i} \omega_{q+G} a_{q+G} \\
& -\mathrm{i} \sqrt{M}\left(\mathcal{G}_{1, q+G}^{*} b_{q}-\mathrm{e}^{-\mathrm{i} G \varrho} \mathcal{G}_{2, q+G}^{*} d_{q}\right), \\
& \dot{b}_{q}=-\mathrm{i} \omega_{1} b_{q}-\mathrm{i} \sqrt{M} \sum_{G} \mathcal{G}_{1, q+G} a_{q+G}, \\
& \dot{d}_{q}=-\mathrm{i} \omega_{2} d_{q}-\mathrm{i} \sqrt{M} \sum_{G} \mathrm{e}^{\mathrm{i} G \varrho} \mathcal{G}_{2, q+G} a_{q+G},
\end{aligned}
$$

where $\omega_{q}=c|q|$. Hence, the spin wave at wavevector $q$ couples in principle with all photonic modes at wavevectors $q+G$. Nevertheless, only the coupling of the atomic transition with the quasi-resonant modes at wavevectors $Q= \pm G_{0}$ is significant. Taking into account only the relevant coupling, we can solve analytically the eigenvalue problem around $q \simeq 0$ in the BZ and assuming $\omega_{1}=\omega_{2}$. In this limit one finds four eigenfrequencies,

$$
\nu_{j, \pm}=\frac{\omega_{Q}+\omega_{1}}{2} \pm \sqrt{\left(\frac{\omega_{Q}-\omega_{1}}{2}\right)^{2}+M \mathcal{G}^{2}\left(1-(-1)^{j} \sqrt{1-\left(\frac{2\left|\mathcal{G}_{1, Q} \mathcal{G}_{2, Q}\right|}{\mathcal{G}^{2}}\right)^{2} \sin ^{2} G_{0} \varrho}\right)}
$$


where $\mathcal{G}=\sqrt{\left|\mathcal{G}_{1, Q}\right|^{2}+\left|\mathcal{G}_{2, Q}\right|^{2}}$ and $j=1,2$. They determine the edges of two photonic bandgaps, one at the frequencies between $\nu_{1,-}$ and $\nu_{2,-}$ and the second between $\nu_{2,+}$ and $\nu_{1,+}$. We note that the bandgap size depends on the interparticle distance $\varrho$ inside the Wigner-Seitz cell but is independent on the number of cells $M$, and thus it is constant in the thermodynamic limit: in fact the quantization volume $V \propto 1 / \sqrt{M}$ (in $1 \mathrm{D}$ ) gives that $\mathcal{G} \propto 1 / \sqrt{M}$, so that the dependence on $M$ in Eq. (22) cancels out.

For $\varrho \rightarrow 0$, in the limit of the monoperiodic array, one finds a single bandgap with size $\Delta \omega=\nu_{1,+}-\nu_{1,-}$. For $\varrho>0(\varrho<a)$ this interval is reduced: a frequency window opens inside the gap, where light is transmitted, and whose width is given by $\Delta \omega_{\varrho}=\nu_{2,+}-\nu_{2,-}$. The size of the two bandgaps is minimum at $\varrho=a / 4$, and it vanishes at this point when $\mathcal{G}_{1, Q}=\mathcal{G}_{2, Q}$. In this specific case, hence, the lattice becomes completely transparent. This is simply understood, considering that the bandgap results from an interference effect due to multiple scattering by all atomic planes, and it hence depends on the phase relations between the fields scattered by each plane. For this specific configuration, where $\varrho=\lambda / 4,3 \lambda / 4$, the phase accumulated due to scattering of the first atom of the cell cancels out with the phase due to scattering by the second atom. As a result, the total phase accumulated from scattering with the two atoms of the cell is zero, and the medium hence behaves as it were completely transparent. Note that a monoperiodic array is also found for $\varrho=a / 2$, when all atoms have the same scattering properties. In this case the periodicity is halved, and the first BZ doubles, $\left[-G_{0}, G_{0}\right]$. The photonic spectra of this system have been discussed in [8].

These analytical results, obtained in a specific parameter regimes, are confirmed by the results of the numerical spectra, which are evaluated from Eqs. (19) by summing over $40 \mathrm{BZs}$. The photonic spectra are shown in Figs. 2]3(a), where the polariton dispersion relation is reported around $q \simeq 0$ for $\varrho=0,0.2 a, 0.4 a$ for several values of $\omega_{1}$, setting $\omega_{1}=\omega_{2}$. The size of the bandgaps $\Delta \omega_{+}=\nu_{2,+}-\nu_{1,+}$ and $\Delta \omega_{-}=\nu_{2,-}-\nu_{1,-}$ as a function of $\varrho$ are displayed in Figs. 2,3](b), showing that the size of the gap is controlled by $\varrho$, and it vanishes at Figures 4 and 5 display the photonic spectra when the atoms composing the Wigner-Seitz cell are of different species, in the case in which both interact with the probe but the resonance frequency of the respective dipolar transition is different. Figure 4 displays the photonic spectrum for the specific case in which one atomic transition is quasiresonant, while the second is far detuned. In this case three photonic bandgaps appear, which vary largely as a function of $\varrho$, and in such a way that while one is minimum, the other two are maximum, and vice versa. Figure 5 displays the case in which the two atoms composing the cell are far detuned from the probe, with detunings of opposite signs. The spectrum is also characterized by three bandgaps.
In this treatment we neglected atomic absorption, however the evaluated bandgaps are significantly larger than the linewidth $\gamma$, and hence they can be experimentally observed. The effect of absorption is considered in the following section in the framework of a semiclassical model, where also finite size effects are accounted for.

\section{PROBE TRANSMISSION SPECTRA}

We now study the response of the biperiodic array of atoms to an external probe, and evaluate probe reflection and transmission as a function of the probe frequency considering the finite size effects. In this section we assume a three-dimensional array of atoms, where $N$ lattice planes are aligned along the $x$ direction.

The coupling of an external probe to the atomic medium is described by the Maxwell equations for the electric field, in the presence of a dielectric in the region of space $0 \leq x \leq L$, with $L=M a$. The equations for a probe at frequency $\omega_{p}$ and wave vector $k_{p}=\omega_{p} / c$, propagating along $x$, read

$$
\left[\partial_{x}^{2}+k_{p}^{2}\right] \mathbf{E}=-4 \pi k_{p}^{2} \mathbf{P}
$$

where $\mathbf{P}(x, t)$ is the medium polarization. Continuity of the field and its spatial derivative at the interfaces imposes

$$
\begin{aligned}
& \mathbf{E}\left(x=0_{+}\right)=\mathbf{E}\left(x=0_{-}\right), \\
& \partial_{z} \mathbf{E}\left(x=0_{-}\right)-\partial_{z} \mathbf{E}\left(x=0_{+}\right)=4 \pi k_{p}^{2} \mathbf{P}(x=0),
\end{aligned}
$$

and similarly at $x=L$. We consider the classical limit of these equations, which is found from the quantum model within an input-output formalism when the probe is a coherent state [9]. In particular, we calculate the reflection and transmission coefficients of an incident field by means of the transfer matrix method.

We consider a probe field propagating at normal incidence at $x=0$ to a single polarizable plane composed by atoms at resonance frequency $\omega_{j}$ and linewidth $\gamma_{j}$. In the linear response regime, $\mathbf{P}=n_{s} \alpha_{j} \delta(x) \mathbf{E}$ in Eqs. (23) and (24), where $n_{s}$ is the surface particle density and

$$
\alpha_{j}=\frac{3}{4 \pi^{2}} \frac{\varepsilon_{0}}{\hbar} \lambda_{p}^{3}\left(\frac{2 \delta_{j} / \gamma_{j}+i}{1+4 \delta_{j}^{2} / \gamma_{j}^{2}}\right)
$$

is the classical polarizability per particle, with $\delta_{j}=$ $\omega_{j}-\omega_{p}$ the detuning with respect to the atomic transition [4]. Denoting by $\mathbf{E}_{\mathbf{0}}$ the amplitude of the incident field, we write the reflected and transmitted fields as $\mathbf{E}_{r}=r_{j} \mathbf{E}_{0} e^{-i k_{p} x}$ and $\mathbf{E}_{t}=t_{j} \mathbf{E}_{0} e^{i k_{p} x}$, where $r_{j}$ and $t_{j}$ are the transmission coefficients at a plane with polarizability $\alpha_{j}$, and read [4]

$$
r_{j}=\frac{i \xi_{j}}{1-i \xi_{j}}, \quad t_{j}=\frac{1}{1-i \xi_{j}},
$$

with $\xi_{j}=2 \pi k_{p} n_{s} \alpha_{j}$. The transfer matrix $\mathcal{M}_{j}$ relates forward- and backward-traveling waves $\mathbf{E}_{t}^{+}, \mathbf{E}_{t}^{-}$on the 


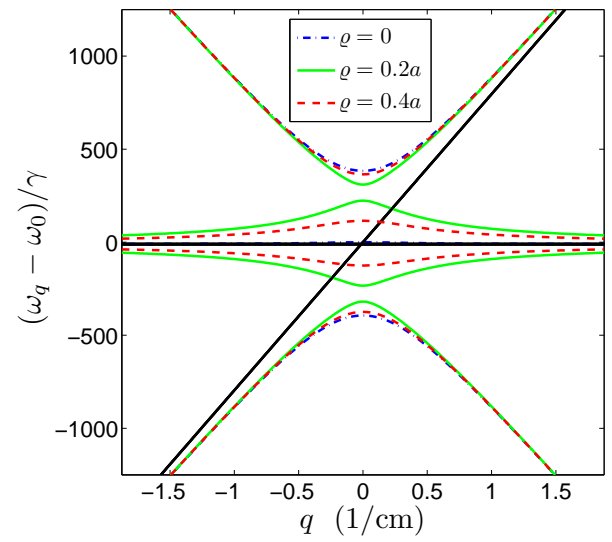

(a)

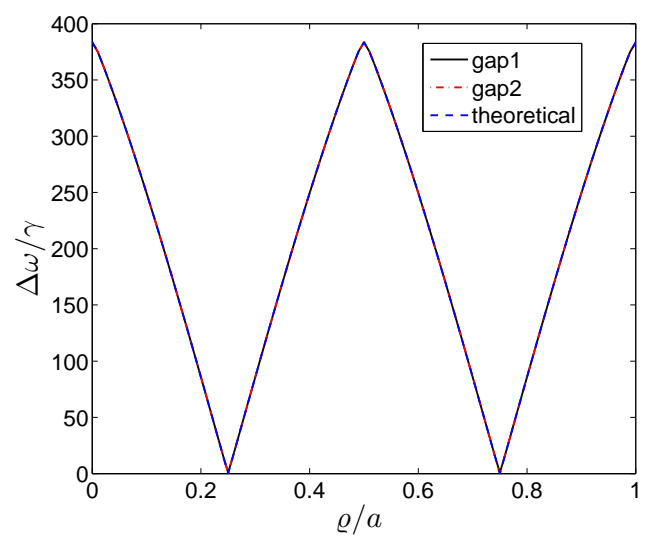

(b)

FIG. 2: (Color online) (a) Polariton dispersion for a 1D bichromatic lattice for different values of $\varrho$ obtained by summing over $40 \mathrm{BZ}$, when the atoms are trapped in a hollow fiber, whose fundamental mode is gaussian with waist $w=5 \mu \mathrm{m}$ 21]. The curves are evaluated for $\omega_{1}=\omega_{2}=\omega_{0}-10 \gamma$, considering the D2-line of ${ }^{85} \mathrm{Rb}$ atoms, with $\lambda=780 \mathrm{~nm}$ and $\gamma=2 \pi \times 6 \mathrm{MHz}$. The solid black line is plotted for comparison, and corresponds to the case in which atoms and field are not coupled $\left(\mathcal{G}_{j}=0\right)$. (b) Photonic bandgap (numbered from lower to higher frequency) as a function of $\varrho / a$, compared with the analytical prediction obtained from Eq. (22).

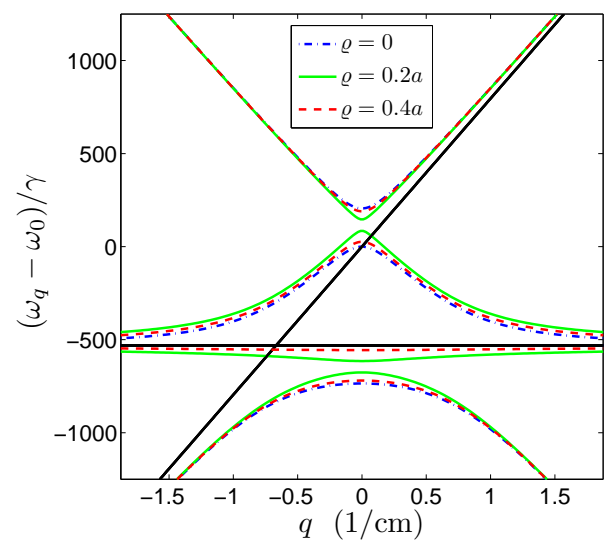

(a)

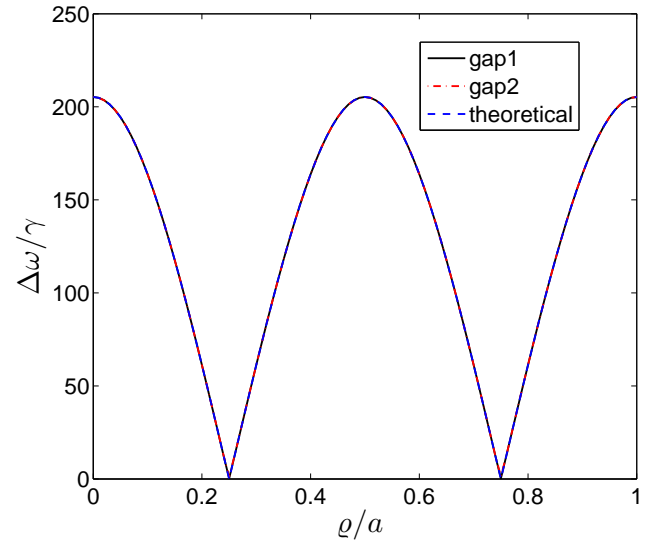

(b)

FIG. 3: Same as in Fig. 2 but for $\omega_{1}=\omega_{2}=\omega_{0}-530 \gamma$.

right-hand side of a plane to those on the left-hand side $\mathbf{E}_{r}^{+}, \mathbf{E}_{r}^{-}$, according to the relation

$$
\left(\begin{array}{l}
\mathbf{E}_{t}^{+} \\
\mathbf{E}_{t}^{-}
\end{array}\right)=\mathcal{M}_{j}\left(\begin{array}{l}
\mathbf{E}_{r}^{+} \\
\mathbf{E}_{r}^{-}
\end{array}\right)
$$

thereby automatically accounting for all interference effects accumulated along the way. The transfer matrix for a single period is given by the product of the transfer matrix across the boundary of an atomic plane and free propagation for a distance $d_{j}$

$$
\mathcal{M}_{d_{j}}=\frac{1}{t_{j}}\left(\begin{array}{cc}
t_{j}^{2}-r_{j}^{2} & r_{j} \\
-r_{j} & 1
\end{array}\right)\left(\begin{array}{cc}
e^{i k_{p} d_{j}} & 0 \\
0 & e^{-i k_{p} d_{j}}
\end{array}\right) .
$$

For any transfer matrix $\tilde{\mathcal{M}}=\prod_{j} \mathcal{M}_{d_{j}}$, generated by the product of single-period transfer matrices, the reflection and the transmission coefficients associated with the matrix elements are

$$
r=\frac{\tilde{\mathcal{M}}_{12}}{\tilde{\mathcal{M}}_{22}}, \quad t=\frac{1}{\tilde{\mathcal{M}}_{22}},
$$

as is easily verified for the special case of matrix in Eq. (28). The transmission and reflection coefficients for the biperiodic lattice of $M=N / 2$ planes is found as a function of the transfer matrix $\mathcal{M}_{d_{1} d_{2}}=\mathcal{M}_{d_{1}} \cdot \mathcal{M}_{d_{2}}$ for a single dimer, with $d_{1}=\varrho, d_{2}=a-\varrho$. In particular, the transmission coefficient reads 


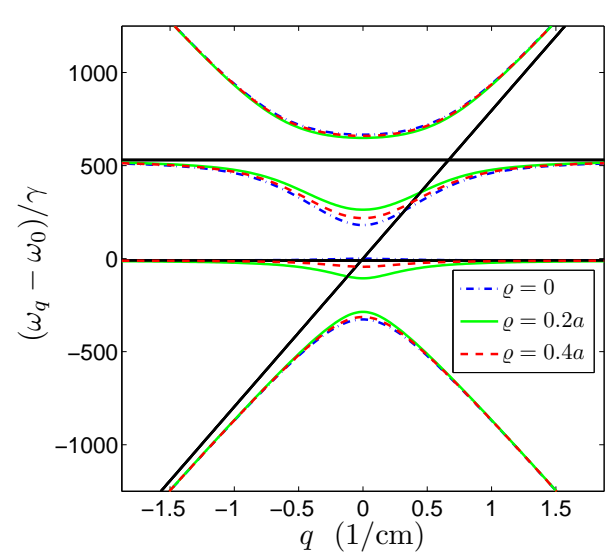

(a)

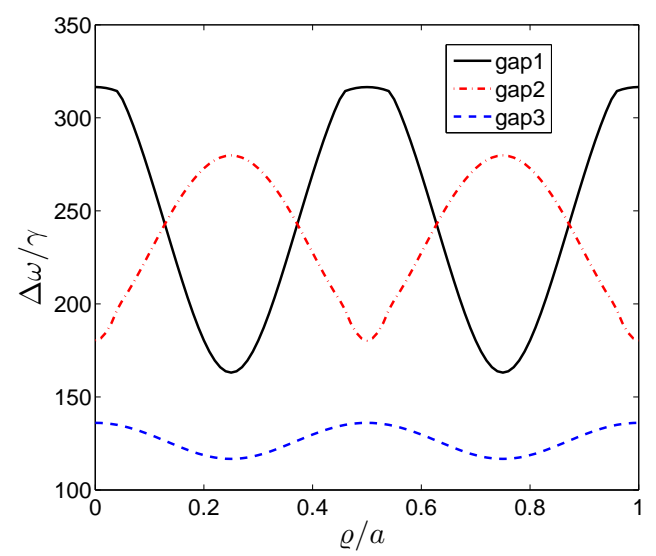

(b)

FIG. 4: Same as in Fig. 2 but for $\omega_{1}=\omega_{0}-10 \gamma$ and $\omega_{2}=\omega_{0}+530 \gamma$.

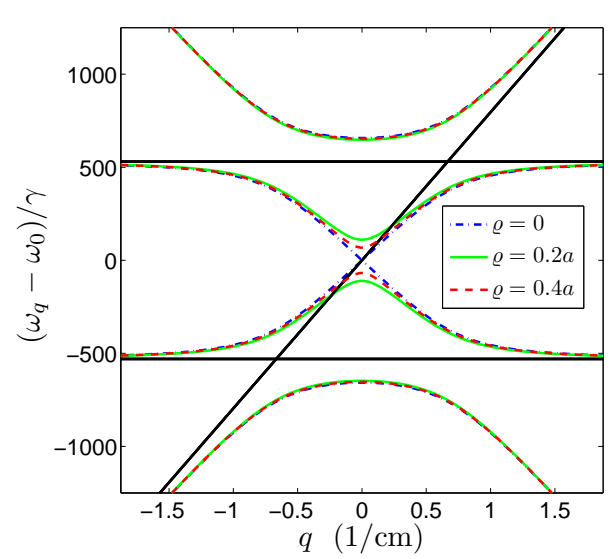

(a)

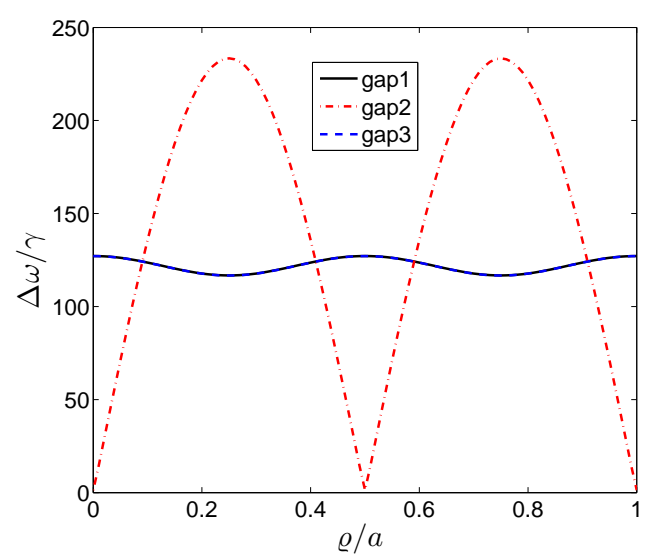

(b)

FIG. 5: Same as in Fig. 2 but for $\omega_{1}=\omega_{0}-530 \gamma$ and $\omega_{2}=\omega_{0}+530 \gamma$. Note that gap 1 and 3 overlap.

$$
t_{n}=\frac{1}{\left(\mathcal{M}_{d_{1} d_{2}}^{n}\right)_{22}}=\frac{\sin \Theta}{\sin \Theta \cos (n \Theta)+i \sin (n \Theta)\left[\xi_{1} \xi_{2} \sin k_{p} \varrho-\left(1-\xi_{1} \xi_{2}\right) \sin k_{p} a-\left(\xi_{1}+\xi_{2}\right) \cos k_{p} a\right]},
$$

with $\Theta=\operatorname{acos}\left[\xi_{1} \xi_{2} \cos k_{p} \varrho+\left(1-\xi_{1} \xi_{2}\right) \cos k_{p} a-\left(\xi_{1}+\xi_{2}\right) \sin k_{p} a\right]$. The elementary cell dephasing $\Theta$ can be written as function of the single slice dephasings $\Theta_{1,2}=\operatorname{acos}\left(\cos k_{p} d_{1,2}-\xi_{1,2} \sin k_{p} d_{1,2}\right)$ as following

$$
\Theta=\operatorname{acos}\left[\cos \left(\Theta_{1}+\Theta_{2}\right)+\sin \Theta_{1} \sin \Theta_{2}-\sqrt{\sin ^{2} \Theta_{1} \sin ^{2} \Theta_{2}-\xi_{1} \xi_{2} \sin ^{2} k_{p} \varrho}\right],
$$

showing that the term $\sin ^{2} k_{p} \varrho$ governs the difference between a simple addition of the dephasings $\Theta_{j}$, as already pointed out in the calculation of the spectrum.

If $\operatorname{Im} \Theta>\operatorname{Re} \Theta$, which occurs around $\omega_{p} \simeq \omega_{1}, \omega_{2}$, in the limit $n \rightarrow \infty$ the transmission coefficient (30) can be approximated by

$$
\lim _{n \rightarrow \infty} t_{n}=\frac{2 e^{-n \operatorname{Im} \Theta} \sin \Theta}{\sin \Theta+\xi_{1} \xi_{2} \sin k_{p} \varrho-\left(1-\xi_{1} \xi_{2}\right) \sin k_{p} a-\left(\xi_{1}+\xi_{2}\right) \cos k_{p} a} .
$$

For vanishing values of $\operatorname{Im} \Theta$, the effect of multiple scat- tering cannot be factorized in a simple attenuation co- 

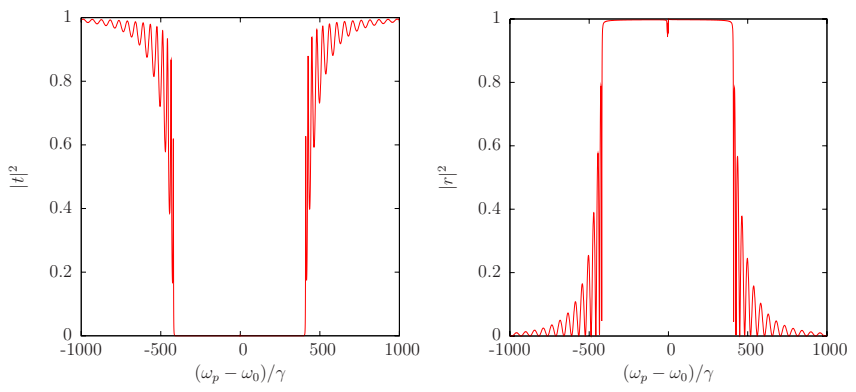

FIG. 6: The absolute square of the transmission coefficient (left panel) and of the reflection coefficient (right panel) as a function of $\left(\omega_{p}-\omega\right) / \gamma$ is plotted for a lattice of ${ }^{85} \mathrm{Rb}$ atoms with areal density $n_{s}=5.7 \cdot 10^{-2} \mu \mathrm{m}^{-2}$, trapped by laser beams detuned to the blue of resonance by $10 \gamma$ and for $10^{6}$ atomic planes.

efficient, and gives rise to interference structures as a function of $\varrho$.

In order to compare the transmission and reflection spectra with the results obtained for the photonic band structure, we consider a cloud of ${ }^{85} \mathrm{Rb}$ atoms in a quasione-dimensional geometry, confined in a very long lattice, taking $N=10^{6}$ atomic slices. We fix the areal density at $n_{s}=5.7 \cdot 10^{-2} \mu \mathrm{m}^{2}$, and we consider that the atoms are in the same internal state, so that $\omega_{2}=\omega_{1}$ and $\gamma_{2}=\gamma_{1}$. In this section we will refer to optical lattices generated by laser beams with a frequency $\omega_{0}=\omega_{1}+10 \gamma$, where $\omega_{1}$ and $\gamma_{1}$ refer to the $D 2$ atomic resonance at $\lambda=780$ $\mathrm{nm}$. Figure 6 displays the absolute square of the transmission coefficient (left panel) and of the reflection coefficient (right panel) of a probe beam traveling through a monoperiodic sequence of atomic planes $(\varrho=0)$. For our choice of the parameters we find the edges of the gap at $\pm 420 \gamma$. The width of the gap depends on our choice for $n_{s}$. The spectrum of transmission agrees qualitatively, as a function of $\varrho$, with the one shown in Fig. 2, where a strictly one-dimensional system was considered. Notice that the small peak in the transmission and the small dip in the reflectivity at the atomic frequency are a mark of scattering losses.

In a bichromatic lattice, such as that shown in Fig. 1. the structure of the transmission and reflection spectrum is deeply modified by the multiperiodicity. Figures 7 and 8 refer to the cases $\varrho=0.2 a$, and $0.24 a$ respectively.

In agreement with the results for the band structure at $\varrho=0.2 a$ in Fig. 2, at the center of the gap it appears a high transmission region divided in two symmetric parts by the absorption line (Fig. 7). By increasing $\varrho$ (Fig. 8), the width of the mini-band increases and the gaps become thinner. At $\varrho=0.25 a$, the gaps close and the atomic lattice becomes transparent in this region of frequencies, everywhere except for $\omega_{p} \simeq \omega_{0}$, because of the absorption. For this particular value of $\varrho, \mathcal{M}_{d_{2}} \simeq \mathcal{M}_{-_{1}} \simeq \mathcal{M}_{d_{1}}^{-1}$, if $\xi_{2}=\xi_{1}$ and neglecting absorption (the imaginary part of $\left.\xi_{j}\right)$.
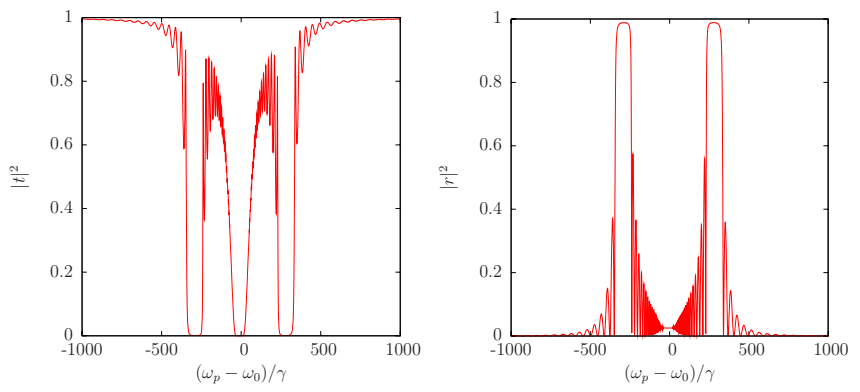

FIG. 7: Same as Fig. 6] but $\varrho=0.2 \lambda$.
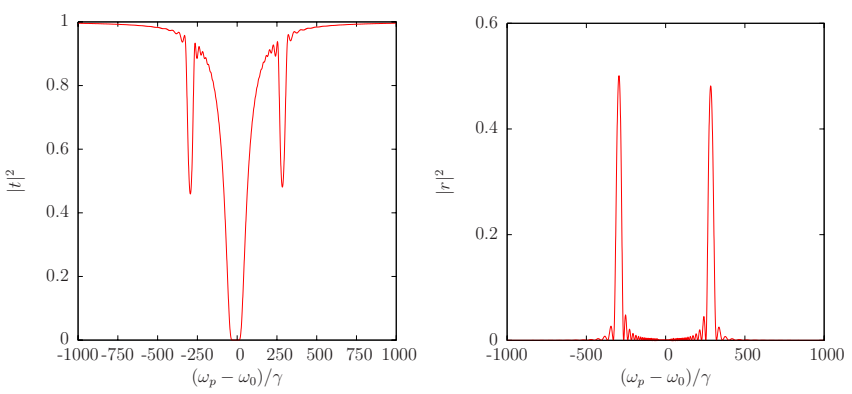

FIG. 8: Same as Fig. 6] but for $\varrho=0.24 \lambda$.

\section{BICHROMATIC LATTICE INSIDE A SINGLE-MODE CAVITY}

The observation of sufficiently large photonic bandgaps in free space requires a large number of lattice planes in a well controlled periodic structure, which is experimentally challenging. Nevertheless, observable effects can be found in small systems when coupling the atomic transition, for instance, to the modes of a fiber [21] or of an optical resonator [23].

Let us assume that the dipolar transition of the atoms couples strongly with the single mode of a standingwave optical resonator, which probes the system. The Hamiltonian for the dynamics inside the cavity is now $H^{\prime}=H_{\text {dip }}+H_{c}+H_{\text {int }}^{\prime}$, where $H_{\text {dip }}$ is given in Eq. (2),

$$
H_{c}=\hbar \omega_{c} a^{\dagger} a
$$

describes the cavity field at frequency $\omega_{c}$, with $a, a^{\dagger}$ annihilation and creation operators of a cavity photon, and

$$
H_{\mathrm{int}}^{\prime}=\hbar \sum_{j} g_{j} \cos \left(k x_{j}+\varphi\right) \sigma_{j}^{\dagger} a+\text { H.c }
$$

is the Jaynes-Cummings Hamiltonian, with $k$ the cavity mode wave vector and $g_{j}$ the coupling strength, which depends on the dipolar moment $\mathcal{D}_{j}$ of the atom at position $x_{j}$. As a function of the cavity parameters, $g_{j}=\sqrt{\varsigma /(4 \pi A)} \sqrt{\gamma \delta \omega}$, with $\varsigma$ the scattering cross section in free space, $A=\pi w_{c}^{2} / 4$ with $w_{c}$ the cavity mode waist, and $\delta \omega=2 \pi c / L$ the free spectral range, with $L$ the cavity length [19]. The phase $\varphi$ accounts for the dephasing between the cavity mode lattice and the atomic lattice. 
The system is probed by an external weak coherent pump at intensity $\eta$ and frequency $\omega_{p}$, which is coupled to the resonator. In this limit, we make the HolsteinPrimakoff transformation and keep only the linear term. The resulting Heisenberg-Langevin equations of motion for cavity mode and spin wave operators read [24]

$$
\begin{aligned}
\dot{a} & =-\mathrm{i} \delta_{c} a-\kappa a+\eta+\sqrt{2 \kappa} \zeta(t)-\mathrm{i} \frac{\sqrt{M}}{2} \\
& \times \sum_{Q \in B Z, Q=k-G}\left[g_{1}\left(\mathrm{e}^{-\mathrm{i} \varphi} b_{Q}+\mathrm{e}^{\mathrm{i} \varphi} b_{-Q}\right),\right. \\
& \left.+g_{2}\left(\mathrm{e}^{-\mathrm{i}(k \varrho+\varphi)} d_{Q}+\mathrm{e}^{\mathrm{i}(k \varrho+\varphi)} d_{-Q}\right)\right] \\
\dot{b}_{ \pm Q} & =-\left(\mathrm{i} \delta_{1}+\frac{\gamma_{1}}{2}\right) b_{ \pm Q}-\frac{\mathrm{i}}{2} \sqrt{M} g_{1} \mathrm{e}^{ \pm \mathrm{i} \varphi} a+\sqrt{\gamma_{1}} \mathcal{B}_{1, Q},
\end{aligned}
$$

whether $\mathcal{N}$ is even or odd, respectively. The eigenfrequencies of the homogeneous equations (for the specific case $\delta_{1}=\delta_{2}=\Delta, \gamma_{1}=\gamma_{2}=\gamma$ ) have the same form as in Eqs. (36]37), whereby now in Eq. (37) the coefficient $\mathcal{R}$ reads

$$
\mathcal{R}=g_{1}^{2} \cos ^{2} \varphi+g_{2}^{2} \cos ^{2}(k \varrho+\varphi) .
$$

We note that the eigenfrequencies in this case explicitly depend on $\varrho$. This result is also found when the bichromatic lattice is replaced by one single cell, by rescaling the coupling strength of each atom inside the cell as $g_{\text {eff }, j}=\sqrt{M} g_{j}$.

We now discuss the intensity of the field at the cavity output as a function of the probe frequency $\omega_{p}$ for various parameters, by solving Eqs. (35a)-(35c) numerically. The (35b) quantity we study is the number of photons per unit time $I\left(\omega_{p}\right)=\left\langle a_{\text {out }}^{\dagger} a_{\text {out }}\right\rangle$, where $a_{\text {out }}$ is the field at the cavity $\dot{d}_{ \pm Q}=-\left(\mathrm{i} \delta_{2}+\frac{\gamma_{2}}{2}\right) d_{ \pm Q}-\frac{\mathrm{i}}{2} \sqrt{M} g_{2} \mathrm{e}^{ \pm \mathrm{i}(k \varrho+\varphi)} a+\sqrt{\gamma_{2}} \mathcal{B}_{2, Q}$

where $\delta_{c}=\omega_{c}-\omega_{p}$ and $\kappa$ is the cavity linewidth. The noise operators $\zeta(t), \mathcal{B}_{j, Q}$, have zero mean value and satisfy the relation $\left\langle\zeta(t) \zeta\left(t^{\prime}\right)^{\dagger}\right\rangle=\left\langle\mathcal{B}_{j, Q}(t) \mathcal{B}_{j, Q}\left(t^{\prime}\right)^{\dagger}\right\rangle=$ $\delta\left(t-t^{\prime}\right)$ (we assume the electromagnetic field in the vacuum).

Equations (35a)-35c describe the coupling between the cavity mode, at momentum $k$, and the spin waves at quasi-momentum $Q$ (inside the first Brillouin zone), such that $Q+G=k$ where $G$ is a vector of the reciprocal lattice. We identify two relevant cases, when (i) $k \neq$ $\mathcal{N} \pi / a$, and (ii) $k=\mathcal{N} \pi / a$, where $\mathcal{N}$ is an integer.

For $k \neq \mathcal{N} \pi / a$ the system, composed by cavity potential and bichromatic lattice, is not periodic. The eigenfrequencies of the homogeneous equations can be simply found for the case $\delta_{1}=\delta_{2}=\Delta, \gamma_{1}=\gamma_{2}=\gamma$, and read

$$
\begin{aligned}
\nu_{0}= & \Delta-\mathrm{i} \gamma / 2 \\
\nu_{ \pm}= & \frac{\delta_{c}+\Delta-\mathrm{i}(\kappa+\gamma / 2)}{2} \\
& \pm \sqrt{\frac{1}{4}\left(\delta_{c}-\Delta-\mathrm{i} \kappa+\mathrm{i} \frac{\gamma}{2}\right)^{2}+M \mathcal{R}}
\end{aligned}
$$

where

$$
\mathcal{R}=\left(g_{1}^{2}+g_{2}^{2}\right) / 2
$$

Here, the real part gives the position of the resonances, while the imaginary part gives the corresponding linewidth. The eigenmodes at frequency $\nu_{0}$ are pure spin waves, and hence correspond to collective dipolar excitations which are decoupled from the cavity field. The eigenmodes at frequency $\nu_{ \pm}$are polariton excitations. We remark that the frequencies $\nu_{ \pm}$do not depend on $\varrho$.

For $k=\mathcal{N} \frac{\pi}{a}$, the system, composed by cavity potential and bichromatic lattice, is periodic. The cavity mode couples to the spin waves $Q=0$ or $\pi / a$, depending on
, output, $a_{\text {out }}=\sqrt{2 \kappa} a-\zeta$, and the average is taken over the state of the system and the vacuum state of the e.m.field outside the resonator [24]. Hence, we find

$$
I\left(\omega_{p}\right)=2 \kappa\left\langle a^{\dagger} a\right\rangle=\frac{2 \kappa \eta^{2}\left(\Delta^{2}+\gamma^{2} / 4\right)}{\left(\kappa \gamma / 2-\delta_{c} \Delta+M \mathcal{R}\right)^{2}+\left(\Delta \kappa+\delta_{c} \gamma / 2\right)^{2}},
$$

where we have used the steady state solution of Eq. (35a). In the strong coupling regime, when the cooperativity $\mathcal{C} \sim M \mathcal{R} / 2 \kappa \gamma \gg 1$ [19], the intensity $I\left(\omega_{p}\right)$ at the cavity output exhibits two well defined maxima at the frequencies

$$
\omega_{p}^{0}=\frac{\omega_{c}+\omega_{a}}{2} \pm \sqrt{\left(\frac{\omega_{c}-\omega_{a}}{2}\right)^{2}+M \mathcal{R}}
$$

and corresponding to the vacuum Rabi splitting for this system [19]. This can also be seen in Figs. 9] and 10, It is interesting to note that for $\Delta=0$ and large cooperativity the cavity output field goes to zero as $1 / \mathcal{C}^{2}$. This is an interference effect, where the atomic polarization inside the cavity form a field equal and opposite to the driving pump, such that the cavity field is effectively empty. Energy is in this case dissipated by the atoms. This behaviour has been first predicted in 25] under the name "cavity induced transparency".

We evaluate the cavity transmission spectrum using the parameters of the setup in [26], and consider ${ }^{85} \mathrm{Rb}$ atoms inside a resonator with length $L=85 \mathrm{~mm}$, finesse $\mathcal{F}=170000$, loss rate $\kappa=2 \pi \times 21 \mathrm{KHz}$, beam waist $w=130 \mu \mathrm{m}$, and an average occupation per site $\bar{n}=3000$, that corresponds to an areal density $n_{s} \simeq 5.7 \cdot 10^{-2} \mu \mathrm{m}^{-2}$. The reflectivity of the cavity mirrors is $|r|^{2} \simeq 1-1.8 \times 10^{-5}$. The transmission spectrum is calculated assuming that $N=200$ planes $(M=100)$ are confined inside the resonator. The positions of the peaks correspond to those predicted by Eq. (41) by taking into account multiple occupancy of the lattice sites rescaling the coupling strengths as $g_{j} \rightarrow \sqrt{\bar{n}} g_{j}$. Figure 9 displays the squared transmission as a function of the probe frequency for the case $\varphi=\pi / 2$ and the values 


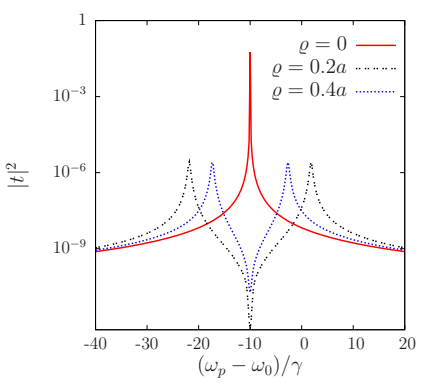

FIG. 9: (Color online) The absolute square of the transmission coefficient as a function of $\left(\omega_{p}-\omega_{0}\right)$ (in units of $\gamma$ ) is plotted for a lattice of ${ }^{85} \mathrm{Rb}$ atoms with areal density $n_{s}=5.7 \cdot 10^{-2} \mu \mathrm{m}^{-2}$, trapped by laser beams detuned to the blue of resonance by $10 \gamma$, for 200 planes, in the presence of a cavity of length $L=85 \mathrm{~mm}$ and finesse $\mathcal{F}=170000$, and for $\varphi=\pi / 2$. Note that for $\varrho=0$ the atoms are trapped at the nodes of the resonator and hence do not interact with the cavity field.

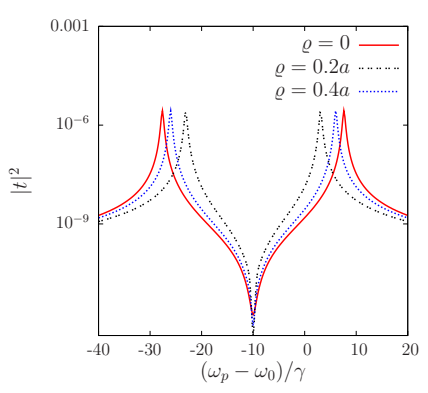

FIG. 10: Same as Fig. 9, but for $\varphi=0$.

$\varrho=0,0.2 a, 0.4 a$. Note that the case $\varrho=0$ corresponds to all atoms at the antinodes of the resonator, and it is hence equivalent to the situation in which the cavity is empty. Figure 10 displays the transmission spectrum is the optical lattice trapping the atom is shifted so that $\varphi=0$, showing that the form changes substantially. By varying $\varphi$, hence, information on the interparticle distance in the Wigner-Seitz cell can be gained. The minimum at $\omega_{p}=\omega_{0}$, corresponding to the cavity induced transparency behaviour, is here visible.

\section{CONCLUSION}

The photonic properties of biperiodic optical lattices are critically determined by the interparticle distance $\varrho$ inside the primitive Wigner-Seitz cell. We have derived a model describing light propagation for a weak probe, and its response to probe propagation in free space and inside of a cavity. We have found that, depending on $\varrho$, in free space the system may or may not exhibit photonic bandgaps about the atomic frequency. This is a peculiar property, which makes the biperiodic crystal different from the monoperiodic one, always exhibiting a bandgap at the atomic frequency. In case there are photonic bandgaps around this value, they occur in two or more ranges of frequencies. For a finite crystal, relevant effects can be observed when the atoms are confined inside an optical resonator. Here, the interparticle distance $\varrho$ inside the primitive Wigner-Seitz cell determines the properties of the transmission spectrum of a probe at the cavity output.

Our study is based on a full quantum model for the light. In this paper we have focused on the intensity of the transmitted and reflected light, in the future we will study higher order coherence of the scattered light. On the basis of studies made with two atoms inside a cavity [27], we expect that, when considering saturation effects, the biperiodic optical lattice can act as nonlinear-optical medium, whose properties may be controlled by the interparticle distance $\varrho$. In the linear response regime, it can be interesting to study higher order coherence of the scattered light for various states of matter inside the potential, with the aim of determining the quantum state of the matter [10]. This may allow, in particular, to detect experimentally novel states of matter realized in bichromatic optical lattices [12, 13, 14].

\section{Acknowledgement}

This work was carried out under the HPC-EUROPA project (RII3-CT-2003-506079), with the support of the European Community - Research Infrastructure Action under the FP6 "Structuring the European Research Area" Programme. Support by the European Commission (EMALI, MRTN-CT-2006-035369; SCALA, Contract No. 015714) and by the Spanish Ministerio de Educación y Ciencia (Consolider Ingenio 2010 QOIT, CSD2006-00019; QNLP, FIS2007-66944; Ramony-Cajal) are acknowledged.
[1] I. Bloch, J. Dalibard, and W. Zwerger, Rev. Mod. Phys. 80, 885 (2008).

[2] M. Lewenstein, A. Sanpera, V. Ahufinger, B. Damski, A. Sen De, U. Sen, Adv. Phys. 56, 243 (2007).

[3] G. Grynberg and C. Robilliard, Phys. Rep. 355, 335
(2001)

[4] I.H. Deutsch, R.J.C. Spreeuw, S.L. Rolston, and W.D. Phillips, Phys. Rev. A 52, 1394 (1995).

[5] D.V. van Coevorden, R. Sprik, A. Tip, and A. Lagendijk, Phys. Rev. Lett. 77, 2412 (1996). 
[6] P. Lambropoulos, G.M. Nikolopoulos, T.R. Nielsen, and S. Bay, Rep. Prog. Phys. 63, 455 (2000).

[7] M. Artoni, G. La Rocca, and F. Bassani, Phys. Rev. E 72, 046604 (2005).

[8] Y.D. Chong, D.E. Pritchard, and M. Soljacic, Phys. Rev. B 75235124 (2007).

[9] F. Bariani and I. Carusotto, J. Eur. Opt. Soc. 3, 08005 (2008).

[10] See for instance I.B. Mekhov, C. Maschler, and H. Ritsch, Nature physics 3, 319 (2007).

[11] P. Zoller et al., Quantum information processing and communication, Eur. Phys. J. D 36, 203 (2005).

[12] N. Bar-Gill, R. Pugatch, E. Rowen, N. Katz, and N. Davidson, preprint arXiv:cond-mat/0603513 (2006).

[13] G. Roati, C. D'Errico, L. Fallani, M. Fattori, C. Fort, M. Zaccanti, G. Modugno, M. Modugno, and M. Inguscio, Nature (London) 453, 895 (2008).

[14] P. Barmettler, A.M. Rey, E. Demler, M.D. Lukin, I. Bloch, and V. Gritsev, Phys. Rev. A 78, 012330 (2008).

[15] S. Fölling, S. Trotzky, P. Cheinet, M. Feld, R. Saers, A. Widera, T. Müller and I. Bloch, Nature 448, 1029 (2007).

[16] J. E. Lye, L. Fallani, C. Fort, V. Guarrera, M. Modugno, D. S. Wiersma, and M. Inguscio, Phys. Rev. A 75, 061603(R) (2007).

[17] O. Mandel, M. Greiner, A. Widera, T. Rom, T. W. Hänsch, and I. Bloch, Phys. Rev. Lett. 91, 010407 (2003).

[18] S. Stenholm, Rev. Mod. Phys. 58, 699 (1986).
[19] H. J. Kimble, in Cavity Quantum Electrodynamics, edited by P. Berman, (Academic, New York, 1994), p. 203.

[20] D.E. Chang, V. Gritsev, G. Morigi, V. Vuletic, M.D. Lukin, and E.A. Demler, Nature physics 4, 884 (2008).

[21] C.A. Christensen, S. Will, M. Saba, G.-B. Jo, Y.-I. Shin, W. Ketterle, D. Pritchard, preprint arXiv:0808.0054v2 (2008).

[22] T. Holstein and H. Primakoff, Phys. Rev. 58, 1098 (1940)

[23] S. Slama, C. von Cube, A. Ludewig, M. Kohler, C. Zimmermann, and Ph. W. Courteille, Phys. Rev. A 72, 031402(R) (2005); S. Slama, C. von Cube, B. Deh, A. Ludewig, C. Zimmermann, and Ph.W. Courteille, Phys. Rev. Lett. 94, 193901 (2005); S. Slama, C. von Cube, M. Kohler, C. Zimmermann, and Ph. W. Courteille, Phys. Rev. A 73, 023424 (2006).

[24] D. F. Walls and G. J. Milburn, Quantum Optics (Springer, Berlin, 1994).

[25] P.R. Rice and R.J. Brecha, Opt. Comm. 126, 230 (1996); for a discussion about the excitation spectrum for large cooperativity see also S. Zippilli, G. Morigi, and H. Ritsch, Eur. Phys. J. D 31, 507 (2004).

[26] D. Kruse, M. Ruder, J. Benhelm, C. von Cube, C. Zimmermann, Ph. W. Courteille, Th. Elsässer, B. Nagorny, and A. Hemmerich, Phys. Rev. A 67, 051802(R) (2003).

[27] S. Fernandez-Vidal, S. Zippilli, and G. Morigi, Phys. Rev. A 76, 053829, (2007). 